Pacific Journal of Mathematic 


\title{
FUNCTIONALS ON CONTINUOUS FUNCTIONS
}

\author{
J. R. BAXter and R. V. Chacon
}

Let $\mathscr{C}(M)$ be the space of continuous functions on a compact metric space $M$. In a previous paper a class of nonlinear functionals $\Phi$ on $\mathscr{C}([0,1] \times[0,1])$ was constructed, such that each $\Phi$ satisfied:

(i) $\lim _{\|f\| \rightarrow 0} \Phi(f)=0$,

(ii) $\Phi(f+g)=\Phi(f)+\Phi(g)$ whenever $f g=0$, and

(iii) $\Phi(f+\alpha)=\Phi(f)+\Phi(\alpha)$ for any constant $\alpha$.

In this paper we show that the dimensionality of $[0,1] \times$ $[0,1]$ is what makes the construction work. More precisely, we show that if $\Phi$ is a functional on $\mathscr{C}(M)$ satisfying (i), (ii), and (iii), and if the dimension of $M$ is less than two, then $\Phi$ must be linear.

1. Introduction. Let $M$ be a compact metric space. Let $\mathscr{C}(M)$ be the space of continuous real-valued functions on $M$. In this paper, we will prove the following result:

THEOREM 1. Let $\Phi: \mathscr{C}(M) \rightarrow \boldsymbol{R}(\boldsymbol{R}=$ the real numbers $)$ be a functional such that:

(i) $\lim _{\|f\| \rightarrow 0} \Phi(f)=0,\left(\|f\|=\sup _{x \in M}|f(x)|\right)$

(ii) $\Phi(f+g)=\Phi(f)+\Phi(g)$ whenever $f g=0$

(iii) $\Phi(f+\alpha)=\Phi(f)+\Phi(\alpha)$ for all $f \in \mathscr{C}(M), \alpha \in \boldsymbol{R}$.

Then if $M$ has dimension no greater than one, $\Phi$ must be linear.

The additivity properties (ii) and (iii) may also be expressed by one condition:

(ii) $\Phi(f+g)=\Phi(f)+\Phi(g)$ whenever $g$ is constant on $\{x \mid f(x) \neq 0\}$.

It is also easy to see that we must have $\Phi(\alpha)=\alpha \Phi(1)$ for all $\alpha \in \boldsymbol{R}$.

It has been shown in [2] that there exist nonlinear functionals $\Phi$ on $\mathscr{C}([0,1] \times[0,1])$ which are bounded, continuous, monotonic, and satisfy conditions (ii) and (iii). Thus Theorem 1 does not extend to spaces of dimension greater than one.

In [1], a proof of Theorem 1 is given for the special case $M=$ $[0,1]$. We will use this case of Theorem 1 to prove the general case. In $\S 2$ it is shown that Theorem 1 is equivalent to the following result:

THEOREM 2. For each $f \in \mathscr{C}(M)$, let $\mathscr{B}_{f}=\left\{f^{-1}(E) \mid E \leqq R, E\right.$ Borel\}. Suppose a measure $\mu_{f}$ on $\mathscr{B}_{f}$ is given, for each $f \in \mathscr{C}(M)$, such that: 

and

(i) the measures $\mu_{f}$ are uniformly bounded in total variation,

(ii) the measures $\mu_{f}$ are consistent, in the sense that if $\mathscr{B}_{f} \subseteq \mathscr{B}_{g}$ then $\mu_{f}=\mu_{g}$ on $\mathscr{B}_{f}$.

Then if $M$ has dimension no greater than one, a measure $\mu$ on the Borel sets of $M$ can be found, which is the common extension of all the $\mu_{f}$.

Theorem 2 is obvious if $M$ is the unit interval, but not if $M$ is the unit circle. Theorem 2 will be proved in $\S 3$.

2. Construction of a set function. For each $f \in \mathscr{C}(M)$, let $\mathscr{L}_{f}$ be the space of continuous functions $g \in \mathscr{C}(M)$ which are measurable with respect to $\mathscr{B}_{f}$. It is easy to see that $g \in \mathscr{L}_{f}$ if and only if $g(x)=g(y)$ whenever $f(x)=f(y)$, and that this means $g$ is of the form $h \circ f$, where $h$ is a continuous function on $\boldsymbol{R}$.

LEMMA 1. $\Phi$ satisfies conditions (i), (ii), and (iii) of Theorem 1 if and only if:

(i) $\Phi$ is bounded, that is, there exists $k$ such that $|\Phi(f)| \leqq k\|f\|$ for all $f \in \mathscr{C}(M)$,

(ii) $\Phi$ is linear on each space $\mathscr{L}_{f}$.

Proof. Assume $\Phi$ satisfies (i), (ii) and (iii) of Theorem 1. Fix $f \in \mathscr{C}(M)$. Let $I$ be a compact interval containing $f(M)$.

Define $\Phi^{*}$ on $\mathscr{C}(I)$ by the equation $\Phi^{*}(h)=\Phi(h \circ f)$. Clearly $\Phi^{*}$ satisfies conditions (i), (ii), and (iii) of Theorem 1. By the special case of Theorem 1 that is proved in [1], $\Phi^{*}$ must be linear. It follows at once that $\Phi$ is linear on $\mathscr{L}_{f}$.

Since $\Phi$ is continuous at 0 , there exists $r>0$ such that

$$
\|f\| \leqq r \text { implies }|\Phi(f)| \leqq 1 .
$$

Then for any $f \in \mathscr{C}(M), f \neq 0$,

$$
|\Phi(f)|=\left|\frac{\|f\|}{r} \Phi\left(\frac{r f}{\|f\|}\right)\right| \leqq \frac{1}{r}\|f\| .
$$

Thus $\Phi$ is bounded.

Now assume $\Phi$ satisfies conditions (i) and (ii) of Lemma 1. Then condition (i) of Theorem 1 clearly holds.

To prove that condition (ii) of Theorem 1 holds, let us first assume that $f$ and $g$ are in $\mathscr{C}(M)$, with $f \geqq 0, g \leqq 0$, and $f g=0$.

Then $f=(f+g) \vee 0$ and $g=(f+g) \wedge 0$, so that $f$ and $g$ are both in $\mathscr{L}_{f+g}$. Hence $\Phi(f+g)=\Phi(f)+\Phi(g)$. 
Now assume that $f \geqq 0, g \geqq 0$, and $f g=0$. Then by the preceding argument $f$ and $g$ are both in $\mathscr{L}_{f-g}$, so again $\Phi(f+g)=\Phi(f)+\Phi(g)$.

Finally, for arbitrary $f$ and $g$ in $\mathscr{C}(M)$ with $f g=0$, let $f_{1}=f \vee 0$, $f_{2}=f \wedge 0, g_{1}=g \vee 0, g_{2}=g \wedge 0$. Then

$$
\begin{aligned}
\Phi(f+g) & =\Phi\left(f_{1}+f_{2}+g_{1}+g_{2}\right) \\
& =\Phi\left(f_{1}+g_{1}\right)+\Phi\left(f_{2}+g_{2}\right) \quad \text { by the first case, } \\
& =\Phi\left(f_{1}\right)+\Phi\left(g_{1}\right)+\Phi\left(f_{2}\right)+\Phi\left(g_{2}\right) \quad \text { by the second case, } \\
& =\Phi\left(f_{1}+f_{2}\right)+\Phi\left(g_{1}+g_{2}\right) \quad \text { by the first case, } \\
& =\Phi(f)+\Phi(g) . \text { Thus condition (ii) of Theorem } 1 \text { holds. }
\end{aligned}
$$

Condition (iii) of Theorem 1 clearly holds, so Lemma 1 is proved.

Using Lemma 1 and the Riesz representation theorem it is easy to see that for each functional $\Phi$ satisfying conditions (i), (ii), and (iii) of Theorem 1 we can find a system of measures $\mu_{f}$ satisfying conditions (i) and (ii) of Theorem 2, and such that $\Phi(f)=\int f d \mu_{f}$ for each $f \in \mathscr{C}(M)$. Conversely, if $\mu_{f}, f \in \mathscr{C}(M)$, is a system of measures satisfying conditions (i) and (ii) of Theorem 2 , then Lemma 1 implies that the functional $\Phi$ defined by $\Phi(f)=\int f d \mu_{f}$ must satisfy conditions (i), (ii), and (iii) of Theorem 1. It follows at once that Theorems 1 and 2 are equivalent.

In what follows we will use both $\Phi$ and the corresponding system of measures $\mu_{f}$.

Lemma 2. Let $f$ and $g$ be in $\mathscr{C}(M)$. Let $K$ be a closed set in $\mathscr{B}_{f} \cap \mathscr{B}_{g}$. Then $\mu_{f}(K)=\mu_{g}(K)$.

Proof. $f(K)$ is a compact set in $\boldsymbol{R}$. It is easy to see that one can find a sequence of continuous functions $h_{n}$ on $\boldsymbol{R}$ such that $0 \leqq$ $h_{n} \leqq 1, h_{n}=1$ on a neighborhood of $f(K), h_{n}=1$ on the support of $h_{n+1}$, and the intersection of the supports of the $h_{n}$ is $f(K)$.

Let $f_{n}=h_{n} \circ f$. Then clearly $0 \leqq f_{n} \leqq 1, f_{n}=1$ on a neighborhood of $K, f_{n}=1$ on the support of $f_{n+1}$, and the intersection of the supports of the $f_{n}$ is $K$.

Let $g_{n}=p_{n} \circ g$ be a sequence having the same properties as the $f_{n}$. Fix $f_{n}$. Then $f_{n}=1$ on a neighborhood, $A$, of $K$. Since the intersection of the supports of the $g_{n}$ is $K$, it follows that for sufficiently large $m$ the support of $g_{m}$ will be contained in $A$. Hence, by choosing subsequences and relabelling, we may assume that, in addition to the properties mentioned above, $f_{n}$ and $g_{n}$ are also such that $f_{n}=1$ on a neighborhood of the support of $g_{n}$, and $g_{n}=1$ on a neighborhood of the support of $f_{n+1}$.

Since the $f_{n}$ are uniformly bounded, and $f_{n} \rightarrow \chi_{k}$ pointwise as 
$n \rightarrow \infty$, we have $\Phi\left(f_{n}\right)=\int f_{n} d \mu_{f} \rightarrow \mu_{f}(K)$ as $n \rightarrow \infty$. Similarly $\Phi\left(g_{n}\right) \rightarrow$ $\mu_{g}(K)$ as $n \rightarrow \infty$. Suppose $\mu_{f}(K)>\mu_{g}(K)$. Choose $\delta>0, \delta<\mu_{f}(K)-$ $\mu_{g}(K)$. For sufficiently large $n$ we must have $\Phi\left(f_{n}\right)>\Phi\left(g_{n}\right)+\delta$. By relabelling we may assume that $\Phi\left(f_{n}\right)>\Phi\left(g_{n}\right)+\delta$ for all $n$.

Let $u_{n}$ be a continuous function on $M$ such that $0 \leqq u_{n} \leqq 1$, $u_{n}=0$ on the support of $g_{n}$, and $u_{n}=1$ on $\left\{x \mid f_{n}(x)<1\right\}$. Let

$$
v_{n}=f_{n}-u_{n} f_{n}-g_{n} \text {. }
$$

It is easy to check that $0 \leqq v_{n} \leqq 1$, and the support of $v_{n}$ is contained in

$$
\left\{x \mid f_{n}(x)=1\right\}-\left\{x \mid g_{n}(x)=1\right\} .
$$

Hence $\Phi\left(-v_{n}+f_{n}\right)=\Phi\left(-v_{n}\right)+\Phi\left(f_{n}\right)$, by the additivity property (ii)' of $\Phi$. That is, $\Phi\left(u_{n} f_{n}+g_{n}\right)=\Phi\left(-v_{n}\right)+\Phi\left(f_{n}\right)$. Since $u_{n} f_{n}=0$ on the support of $g_{n}$, we have $\Phi\left(u_{n} f_{n}+g_{n}\right)=\Phi\left(u_{n} f_{n}\right)+\Phi(g)$ by the additivity of $\Phi$ again. Thus $\Phi\left(u_{n} f_{n}\right)+\Phi\left(g_{n}\right)=\Phi\left(-v_{n}\right)+\Phi\left(f_{n}\right)$. Hence $\Phi\left(u_{n} f_{n}\right)>$ $\Phi\left(-v_{n}\right)+\delta$, and so $\sum_{n=1}^{m} \Phi\left(u_{n} f_{n}\right)>\sum_{n=1}^{m} \Phi\left(-v_{n}\right)+m \delta$, for all $m$.

It is easy to check that the supports of the $u_{n} f_{n}$ are pairwise disjoint, as are the supports of the $v_{n}$. Hence

$$
\Phi\left(\sum_{n=1}^{m} u_{n} f_{n}\right)>\Phi\left(\sum_{n=1}^{m}\left(-v_{n}\right)\right)+m \delta,
$$

by additivity, for all $m$.

The functions $\sum_{n=1}^{m} u_{n} f_{n}$ and $\sum_{n=1}^{m}\left(-v_{n}\right)$ are uniformly bounded in $m$. Hence the last inequality contradicts the boundedness of $\Phi$. Hence our original supposition, $\mu_{f}(K)>\mu_{g}(K)$, was false. This proves Lemma 2.

Since $M$ is a metric space, it is easy to see that every closed set $E$ and every open set $E$ occurs in some $\mathscr{B}_{f}$.

Definition 1. Let us write $\mu_{f}(E)=\mu(E)$ for $E$ closed or $E$ open, since the number has been shown to be independent of $f$.

LEMмA 3. The set function $\mu$ is bounded and additive wherever defined.

Proof. $\mu$ is bounded because the total variation of the $\mu_{f}$ 's is uniformly bounded.

Let $E_{1}$ and $E_{2}$ be sets, with $E_{1} \cap E_{2}=\phi$, such that $\mu\left(E_{1}\right), \mu\left(E_{2}\right)$, and $\mu\left(E_{1} \cup E_{2}\right)$ are defined. We may have $E_{1}, E_{2}$ open, $E_{1}, E_{2}$ closed, $E_{1}$ open, $E_{2}$ closed, and $E_{1} \cup E_{2}$ open, or $E_{1}$ open, $E_{2}$ closed, and $E_{1} \cup E_{2}$ closed. In each of the four possible cases it is easy to find a function $f \in \mathscr{C}(M)$ such that $E_{1}$ and $E_{2}$ are in $\mathscr{B}_{f}$. This proves Lemma 3. 
Lemma 4. Let $G_{n}$ be a monotone increasing sequence of open sets, with union $G$. Let $F_{n}$ be a sequence of closed sets such that $G_{n} \leqq F_{n} \leqq G$ for all $n$. Then $\mu\left(G_{n}\right) \rightarrow \mu(G)$ and $\mu\left(F_{n}\right) \rightarrow \mu(G)$ as $n \rightarrow \infty$.

Proof. Suppose $\mu\left(G_{n}\right) \nrightarrow \mu(G)$ or $\mu\left(F_{n}\right) \nrightarrow \mu(G)$. Then there exists a $\delta>0$ and a subsequence $n_{j}$ such that

$$
\left|\mu\left(G_{n_{j}}\right)-\mu(G)\right|+\left|\mu\left(F_{n_{j}}\right)-\mu(G)\right|>\hat{o}
$$

for all $j$. Since the $F_{n}$ are compact we can choose $n_{j}$ so that $F_{n_{j}} \subseteq G_{n_{j+1}}$. It is then a straightforward matter to construct $f \in \mathscr{C}(M)$ such that $G_{n_{j}}, E_{n_{j}} \in \mathscr{B}_{f}$ for all $j$. This contradiction proves the lemma.

3. Proof of the theorems. In this section we will prove:

THEOREM 3. Let $\mu$ be a real-valued set function defined for closed subsets and for open subsets of $M$, such that:

(i) $\mu$ is bounded and additive wherever defined, and

(ii) $\mu$ has the continuity property described in Lemma 4.

Then if $M$ has dimension no greater than one, $\mu$ can be extended to a measure on the Borel sets of $M$.

We can apply Theorem 3 to the set function $\mu$ constructed in the previous section. The Borel measure $\hat{\mu}$ which is an extension of $\mu$ agrees with each measure $\mu_{f}$ on all closed sets in $\mathscr{B}_{f}$. Since each $\mu_{f}$ is obviously regular, $\hat{\mu}$ must be an extension of $\mu_{f}$. Thus Theorem 2 is proved, and hence Theorem 1 also.

From now on let $\mu$ be any set function satisfying conditions (i) and (ii) of Theorem 3.

LEMMA 5. Let $F_{n}$ be a monotone decreasing sequence of closed sets, having intersection $F$. Let $G_{n}$ be a sequence of open sets such that $F_{n} \supseteqq G_{n} \supseteqq F$ for all $n$. Then $\mu\left(F_{n}\right) \rightarrow \mu(F)$ and $\mu\left(G_{n}\right) \rightarrow \mu(F)$ as $n \rightarrow \infty$.

Proof. Follows from condition (ii) by taking complements and using the additivity property.

Definition 2. For any set $E \subseteq M$, define

$$
\nu(E)=\sup \{\mu(F) \mid F \subseteq E, F \text { closed }\} .
$$

Since $\mu$ is bounded, so is $\nu$. Clearly $\nu$ is monotone.

LEMMA 6. Let $E_{1}$ and $E_{2}$ be disjoint subsets of $M$. Then $\nu\left(E_{1} \cup\right.$ $\left.E_{2}\right) \geqq \nu\left(E_{1}\right)+\nu\left(E_{2}\right)$. If $E_{1}$ and $E_{2}$ are either both open or both closed, 
then $\nu\left(E_{1} \cup E_{2}\right)=\nu\left(E_{1}\right)+\nu\left(E_{2}\right)$.

Proof. Follows from the additivity of $\mu$.

Lemma 7. Let $G$ be open. Then

$$
\nu(G)=\sup \{\mu(H) \mid H \subseteq G, H \text { open }\} .
$$

Proof. Follows from the continuity of $\mu$.

We pause now for a general topological lemma.

LEMma 8. Let $X$ be a locally compact separable metric space of dimension 0 . Then $X$ is a countable union of monotone increasing sets that are both compact and open.

Proof. From the definition of dimension 0, each point $x$ has arbitrarily small neighborhoods $G_{x}$ which are both closed and open.

By choosing $G_{x}$ small enough, it can therefore be made both compact and open.

Since $X=\mathrm{U}_{x \in X} G_{x}$, and $X$ has a countable base, we can find $x_{1}, x_{2}, \cdots$ such that $X=\bigcup_{n=1}^{\infty} G_{x_{n}}$. Let $K_{n}=\bigcup_{j=1}^{n} G_{x_{j}}$. Then each $K_{n}$ is both compact and open, and $K_{n} \uparrow X$.

Now we return to $M, \mu$, and $\nu$.

Lemma 9. Let $G$ be open. Let $E$ be open, $E \subseteq G$, such that $\partial E \cap G$ has dimension 0 . Then $\mu(G) \leqq \nu(E)+\nu(G-E)$.

Proof. Let $D=\partial E \cap G$. Let $H=G-\bar{E}$. Then the sets $E, D$, and $H$ are mutually disjoint, and $G=E \cup D \cup H$.

Since $D$ is a closed subset of the locally compact separable metric space $G, D$ is a locally compact separable metric space also.

By Lemma 8, we can find sets $K_{n}$ which are both compact and open in $D$, such that $K_{n} \uparrow D$.

Let $K_{n}=A_{n} \cap D$, where $A_{n}$ is open. Since $K_{n}$ is compact we may choose $A_{n}$ such that $\bar{A}_{n} \subseteq G$. By taking unions if necessary we may choose the $A_{n}$ to be increasing.

Let $E_{n}$ and $H_{n}$ be open sets such that $\bar{E}_{n} \subseteq E, \bar{H}_{n} \subseteq H$ for all $n$, $E_{n} \uparrow E$ and $H_{n} \uparrow H$. Let $G_{n}=E_{n} \cup A_{n} \cup H_{n}$. Then $G_{n}$ is open, $\bar{G}_{n} \subseteq G$, and $G_{n} \uparrow G$. Then $\mu\left(G_{n}\right) \rightarrow \mu(G)$ as $n \rightarrow \infty$, by continuity.

$$
\begin{aligned}
& \text { But for all } n, G_{n}=\left(G_{n} \cap E\right) \cup\left(G_{n} \cap D\right) \cup\left(G_{n} \cap H\right) \\
& =\left(G_{n} \cap E\right) \cup K_{n} \cup\left(G_{n} \cap H\right) \text {. }
\end{aligned}
$$


Thus $\mu\left(G_{n}\right)=\mu\left(G_{n} \cap E\right)+\mu\left(K_{n}\right)+\mu\left(G_{n} \cap H\right)$, by additivity,

$$
\begin{aligned}
& \leqq \nu\left(G_{n} \cap E\right)+\nu\left(K_{n}\right)+\nu\left(G_{n} \cap H\right) \\
& \leqq \nu(E)+\nu(D)+\nu(H) \leqq \nu(E)+\nu(G-E) .
\end{aligned}
$$

This proves Lemma 9.

Lemma 10. Let $G$ be an open set. Let $E$ be open, $E \subseteq G$, such that $\partial E \cap G$ has dimension 0. Then $\nu(G)=\nu(E)+\nu(G-E)$.

Proof. Let $\varepsilon>0$ be given. Choose $H$ open, $H \subseteq G$, such that $\mu(H) \geqq \nu(G)-\varepsilon$. This is possible by Lemma 7 .

Then $\partial(E \cap H) \cap H=\partial E \cap H \subseteq \partial E \cap G$. Hence $\partial(E \cap H) \cap H$ has dimension 0. By Lemma 7, $\mu(H) \leqq \nu(E \cap H)+\nu(H-E \cap H) \leqq$ $\nu(E)+\nu(G-E)$. Hence $\nu(G) \leqq \nu(E)+\nu(G-E)$.

The reverse inequality holds by Lemma 6, so Lemma 10 is proved.

From now on in this section, let $M$ have dimension at most one.

Lemma 11. Let $G_{1}$ and $G_{2}$ be open, with union $G$. Then $\nu(G) \leqq$ $\nu\left(G_{1}\right)+\nu\left(G_{2}\right)$.

Proof. $G_{1}-G_{2}$ and $G_{2}-G_{1}$ are disjoint and relatively closed in $G$. $G$ is a separable metric space of dimension no larger than 1 . Hence by Theorem 1 in [3], section 27II, page 290, we can find an open set $E \subseteq G$ such that $E \supseteqq G_{1}-G_{2}, \bar{E} \cap\left(G_{2}-G_{1}\right)=\varnothing$, and $\partial E \cap G$ has dimension 0 .

By Lemma 10,

$$
\nu(G)=\nu(E)+\nu(G-E) \leqq \nu\left(G_{1}\right)+\nu\left(G_{2}\right) .
$$

LEMMA 12. Let $G_{n}$ be a sequence of open sets. Let $G=\bigcup_{n=1}^{\infty} G_{n}$. Then $\nu(G) \leqq \sum_{n=1}^{\infty} \nu\left(G_{n}\right)$.

Proof. Let $\varepsilon>0$ be given. Choose $F$ closed, $F \cong G$ such that $\mu(F) \geqq \nu(G)-\varepsilon$.

Then there exists $n$ such that $F \subseteq \bigcup_{j=1}^{n} G_{j}$. Hence $\sum_{j=1}^{\infty} \nu\left(G_{j}\right) \geqq$ $\sum_{j=1}^{n} \nu\left(G_{j}\right) \geqq \nu\left(\bigcup_{j=1}^{n} G_{j}\right)$, by Lemma 11 , $\geqq \mu(F)$ by definition.

This proves Lemma 12.

Definition 3. For any set $E \subseteq M$, define $\nu^{*}(E)=\inf \{\nu(G) \mid E \leqq$ $G, G$ open\}. Clearly $\nu^{*}(E)=\nu(E)$ when $E$ is open.

LEMMA 13. $\nu^{*}$ is an outer measure.

Proof. Follows from Lemma 12. 
Lemma 14. Every open set is measurable with respect to $\nu^{*}$, in the sense of Caratheodory.

Proof. Let $G$ be open. Let $E$ be any set. We know

$$
\nu^{*}(E) \leqq \nu^{*}(E \cap G)+\nu^{*}(E-G),
$$

since $\nu^{*}$ is an outer measure. We must show that

$$
\nu^{*}(E) \geqq \nu^{*}(E \cap G)+\nu^{*}(E-G) .
$$

Choose any open set $H$ such that $E \subseteq H$. Let $\varepsilon>0$ be given. Choose $F$ closed, $F \leqq G \cap H$, such that $\nu(F) \geqq \nu(G \cap H)-\varepsilon$. Then $\nu(H) \geqq \nu(F)+\nu(H-F)$, by Lemma 6 , $\geqq \nu(G \cap H)-\varepsilon+\nu(H-F) \geqq$ $\nu^{*}(E \cap G)-\varepsilon+\nu^{*}(E-G)$ by definition.

Hence $\nu(H) \geqq \nu^{*}(E \cap G)+\nu^{*}(E-G)$. By definition, then, $\nu^{*}(E) \geqq$ $\nu^{*}(E \cap G)+\nu^{*}(E-G)$, and Lemma 14 is proved.

Because of Lemma 14 we know that $\nu^{*}$ defines a measure on a $\sigma$-algebra of sets that includes the Borel sets of $M$.

Proof of Theorem 3. First suppose that $\mu$ is nonnegative. Let $G$ be open. By Lemma $7, \mu(G) \leqq \nu(G)$. On the other hand, for any closed subset $F$ of $G, \mu(F) \leqq \mu(F)+\mu(G-F)=\mu(G)$. Thus $\mu(G)=$ $\nu(G) . \quad \nu^{*}$ is a measure on the Borel sets of $M$ which agrees with $\mu$ on open sets and hence on all sets in the domain of $\mu$.

Now let $\mu$ be arbitrary. Consider the set function $\omega=\nu^{*}-\mu$, defined for closed subsets of $M$ and for open subsets of $M$. $\omega$ is nonnegative by Lemma 7. By what has already been proved, $\omega$ can be extended to a Borel measure. But then $\mu=\nu^{*}-\omega$ can be extended also, so the theorem is proved.

\section{REFERENCES}

1. J. R. Baxter and R. V. Chacon, Almost linear operators and functionals on $\mathscr{C}([0,1])$, to appear in Proc. Amer. Math. Soc.

2. - Nonlinear functionals on $\mathscr{C}([0,1] \times[0,1])$, Pacific. J. Math., 48 (1973), 347-353.

3. K. Kuratowski, Topology, Vol. 1, Academic Press, New York, 1968.

Received January 19, 1973.

UNIVERSITY OF BRITISH COLUMBIA 


\section{PACIFIC JOURNAL OF MATHEMATICS}

\section{EDITORS}

RICHARD ARENS (Managing Editor)

University of California

Los Angeles, California 90024
J. DUGUNDJI*

Department of Mathematics

University of Southern California

Los Angeles, California 90007

D. Gilbarg and J. Milgram

Stanford University

Stanford, California 94305
University of Washington

Seattle, Washington 98105

ASSOCIATE EDITORS
E. F. BECKENBACH
B. H. NeumanN
F. WoLF
K. YosHIDA

\section{SUPPORTING INSTITUTIONS}

\author{
UNIVERSITY OF BRITISH COLUMBIA \\ CALIFORNIA INSTITUTE OF TECHNOLOGY \\ UNIVERSITY OF CALIFORNIA \\ MONTANA STATE UNIVERSITY \\ UNIVERSITY OF NEVADA \\ NEW MEXICO STATE UNIVERSITY \\ OREGON STATE UNIVERSITY \\ UNIVERSITY OF OREGON \\ OSAKA UNIVERSITY
}

\author{
UNIVERSITY OF SOUTHERN CALIFORNIA \\ STANFORD UNIVERSITY \\ UNIVERSITY OF TOKYO \\ UNIVERSITY OF UTAH \\ WASHINGTON STATE UNIVERSITY \\ UNIVERSITY OF WASHINGTON
* * * *
AMERICAN MATHEMATICAL SOCIETY \\ NAVAL WEAPONS CENTER
}

The Supporting Institutions listed above contribute to the cost of publication of this Journal, but they are not owners or publishers and have no responsibility for its content or policies.

Mathematical papers intended for publication in the Pacific Journal of Mathematics should be in typed form or offset-reproduced, (not dittoed), double spaced with large margins. Underline Greek letters in red, German in green, and script in blue. The first paragraph or two must be capable of being used separately as a synopsis of the entire paper. Items of the bibliography should not be cited there unless absolutely necessary, in which case they must be identified by author and Journal, rather than by item number. Manuscripts, in duplicate if possible, may be sent to any one of the four editors. Please classify according to the scheme of Math. Rev. Index to Vol. 39. All other communications to the editors should be addressed to the managing editor, or Elaine Barth, University of California, Los Angeles, California, 90024.

100 reprints are provided free for each article, only if page charges have been substantially paid. Additional copies may be obtained at cost in multiples of 50 .

The Pacific of Journal Mathematics is issued monthly as of January 1966. Regular subscription rate: $\$ 72.00$ a year (6 Vols., 12 issues). Special rate: $\$ 36.00$ a year to individual members of supporting institutions.

Subscriptions, orders for back numbers, and changes of address should be sent to Pacific Journal of Mathematics, 103 Highland Boulevard, Berkeley, California, 94708.

PUBLISHED BY PACIFIC JOURNAL OF MATHEMATICS, A NON-PROFIT CORPORATION

Printed at Kokusai Bunken Insatsusha (International Academic Printing Co., Ltd.), 270, 3-chome Totsuka-cho, Shinjuku-ku, Tokyo 160, Japan.

* C. R. DePrima California Institute of Technology, Pasadena, CA 91109, will replace J. Dugundji until August 1974.

Copyright (C) 1973 by Pacific Journal of Mathematics

Manufactured and first issued in Japan 


\section{Pacific Journal of Mathematics}

\section{Vol. 51, No. $2 \quad$ December, 1974}

Robert F. V. Anderson, Laplace transform methods in multivariate spectral theory .................................................. 339

William George Bade, Two properties of the Sorgenfrey plane . . . . . . . . . . . . 349

John Robert Baxter and Rafael Van Severen Chacon, Functionals on continuous

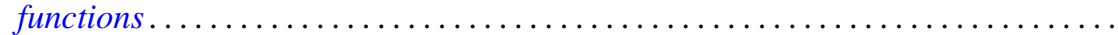

Phillip Wayne Bean, Helly and Radon-type theorems in interval convexity

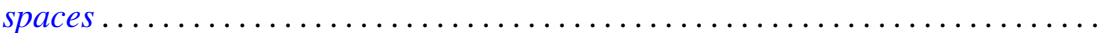

James Robert Boone, On k-quotient mappings $\ldots \ldots \ldots \ldots \ldots \ldots \ldots \ldots \ldots$

Ronald P. Brown, Extended prime spots and quadratic forms . . . . . . . . . . . .

William Hugh Cornish, Crawley's completion of a conditionally upper continuous lattice .............................................

Robert S. Cunningham, On finite left localizations ...................

Robert Jay Daverman, Approximating polyhedra in codimension one spheres

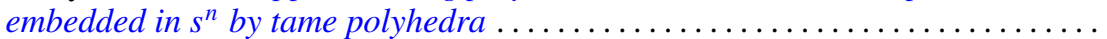

Burton I. Fein, Minimal splitting fields for group representations . . . . . . . . . . . .

Peter Fletcher and Robert Allen McCoy, Conditions under which a connected

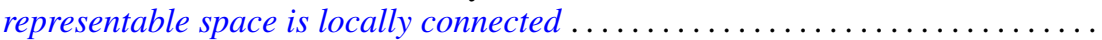

Jonathan Samuel Golan, Topologies on the torsion-theoretic spectrum of a noncommutative ring...

Manfred Gordon and Edward Martin Wilkinson, Determinants of Petrie matrices.

Alfred Peter Hallstrom, A counterexample to a conjecture on an integral condition for determining peak points (counterexample concerning peak points)........

E. R. Heal and Michael Windham, Finitely generated $F$-algebras with applications to Stein manifolds.

Denton Elwood Hewgill, On the eigenvalues of a second order elliptic operator in an unbounded domain ............................

Charles Royal Johnson, The Hadamard product of $A$ and $A^{*}$.

Darrell Conley Kent and Gary Douglas Richardson, Regular completions of Cauchy spaces.

Alan Greenwell Law and Ann L. McKerracher, Sharpened polynomial approximation

Bruce Stephen Lund, Subalgebras of finite codimension in the algebra of analytic functions on a Riemann surface. .

Robert Wilmer Miller, TTF classes and quasi-generators . .

Roberta Mura and Akbar H. Rhemtulla, Solvable groups in which every maximal partial order is isolated ....

Isaac Namioka, Separate continuity and joint continuity...

Alan Saleski, Entropy of self-homeomorphisms of statistical pseudo-metric

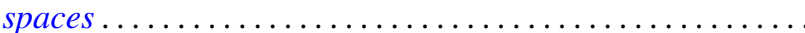

H. A. Seid, Cyclic multiplication operators on $L_{p}$-spaces .....

H. B. Skerry, On matrix maps of entire sequences ............

John Brendan Sullivan, A proof of the finite generation of invariants of a normal

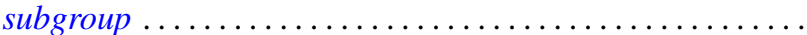

John Griggs Thompson, Nonsolvable finite groups all of whose local subgroups are

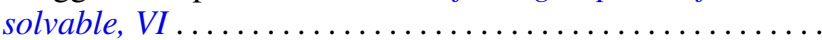

